\title{
Stratified Sadovskii flow in a channel
}

\author{
By S. CHERNYSHENKO \\ Institute of Mechanics, Moscow University, 117192 Moscow, Russia
}

(Received 3 January 1992 and in revised form 30 November 1992)

Stably stratified and non-stratified flows past a touching pair of vortices with continuous velocity are considered. An asymptotic solution for the very long eddies is determined. Numerical results cover the whole range of subcritical stratification and eddy length.

\section{Introduction}

Sadovskii flows are steady inviscid flows past a touching pair of vortex regions, having potential flow far upstream and with constant vorticity of opposite signs inside. Sadovskii (1971 $a$ ) and Saffman \& Tanveer (1982) calculated an unbounded nonstratified flow of this kind and Turfus (1993) made several calculations for the corresponding channel flow. Pierrehumbert (1980) and Smith (1986) considered closely related problems. Sadovskii flows are important because of their role in the asymptotic theory of the steady high-Reynolds-number flow past a bluff body (Chernyshenko 1988).

The generalization to stratified flow in this paper is restricted to the case of a uniform density profile far upstream, constant density inside the eddy equal to the density on the eddy boundary outside, and the Boussinesq approximation throughout.

\section{Problem formulation}

Let us consider the symmetry line dividing the eddy as a lower wall of a channel. Then there is only one region of closed streamlines (see figure 1). Using the channel width as the lengthscale and velocity far upstream as the velocity scale, the equation for the non-dimensionalized streamfunction $\psi$ may be written in the following form:

$$
\left.\begin{array}{rl}
\nabla^{2} \psi & =-\omega(\psi, y), \\
\psi>0, \quad \omega(\psi, y) & =\operatorname{Ri}(\psi-y), \\
\psi<0, \quad \omega(\psi, y) & =-\Lambda=\text { const. }
\end{array}\right\}
$$

Here $A$ is a parameter, $R i$ is the Richardson number, $R i=(\delta \rho / \rho) g H / U^{2}$ where $\rho$ is the density, $\delta \rho$ is the difference between the density at the lower and upper walls, $g$ is the gravitational acceleration and $H$ and $U$ are the dimensional channel height and velocity far upstream, respectively. The density inside the eddy is assumed to be constant and it is continuous at the eddy boundary.

The boundary conditions are

$$
\begin{aligned}
& y=0, \quad \psi=0, \\
& y=1, \quad \psi=1, \\
& x \rightarrow \pm \infty, \quad \psi \rightarrow y .
\end{aligned}
$$




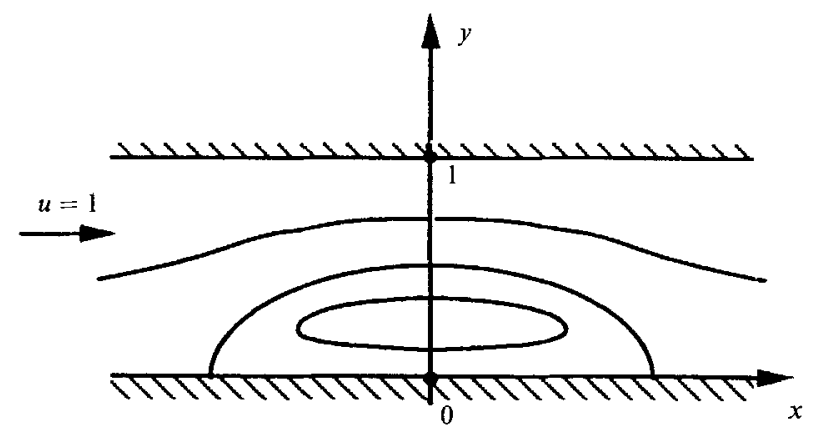

Figure 1. Sketch of the flow (not to scale).

The decrease of the Bernoulli constant on the eddy boundary is taken to be zero because this is a case of interest in the asymptotic theory of the steady high-Reynoldsnumber flow past a bluff body. For the unbounded flow, solutions for $R i=0$ and different values of $A$ are really the same, being different in size only. For the flow in a channel a family of solutions exists. With a fixed upstream velocity an increase in $A$ reduces the size of the eddy and, correspondingly, the influence of the walls on the eddy properties decreases. The appropriate Richardson number must in this case be calculated via the eddy size rather than the channel width and it tends to zero with the eddy size. Eventually for $A \rightarrow \infty$ the unbounded non-stratified Sadovskii flow emerges.

A decrease in $A$ leads to growth of the eddy. The eddy width is limited by the channel walls and the ultimate behaviour is difficult to anticipate but the numerical results described in $\$ 4$ give strong evidence that the eddy width remains finite and the eddy length tends to infinity when $A$ approaches some finite value which depends on $R i$. This implies the long-eddy asymptotics which follow in $\S 3$.

\section{Flows with very long eddies}

When the eddy length $L$ tends to infinity, the proper scale for the longitudinal coordinate $x$ is $L$. Hence let $x=L X, L \rightarrow \infty, X \sim 1$. The asymptotic solution in this limit clearly cannot be valid in the vicinity of the front and rear stagnation points, where a separate analysis is needed. Equation (2.1) may be rewritten as

$$
\frac{1}{L^{2}} \frac{\partial^{2} \psi}{\partial X^{2}}+\frac{\partial^{2} \psi}{\partial y^{2}}=-\omega(\psi, y)
$$

For $L \rightarrow \infty$ we assume $A \rightarrow \Lambda_{\infty}$ and denote $\Psi=\lim _{L \rightarrow \infty} \psi$. In this limit we obtain

$$
\partial^{2} \Psi / \partial y^{2}=-\omega(\Psi, y) .
$$

Together with boundary conditions at the channel walls this gives the following dependence of $\Psi$ on $y$ for a given value of $\Lambda_{\infty}$ :

$$
\left.\begin{array}{cc}
\Psi=y-W_{\infty} \sin \left[(y-1) R i^{\frac{1}{2}}\right] / \sin \left[\left(W_{\infty}-1\right) R i^{\frac{1}{2}}\right], & y>W_{\infty}, \\
\Psi=\frac{1}{2} \Lambda_{\infty}\left(y^{2}-W_{\infty} y\right), & y<W_{\infty},
\end{array}\right\}
$$

where $W_{\infty}$ is the eddy width. The value of $\Lambda_{\infty}$ is still unknown. As $\Psi$ does not depend on $X$, the eddy boundary is flat except in the regions near the eddy ends where the proper lengthscale in both directions is the channel width. Therefore equation (2.1) 


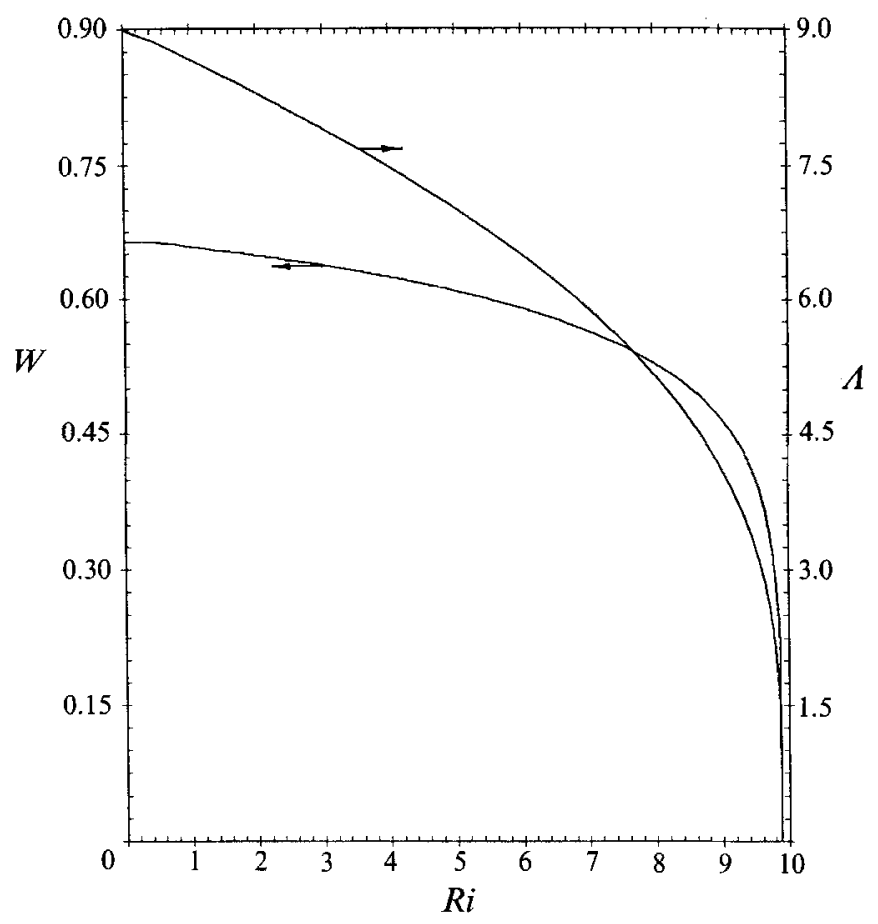

FIGURE 2. The long-eddy asymptotic results.

does not change. The new features of this flow are due to the different upstream and downstream conditions. For the rear end of the eddy, for example, the downstream condition, $x \rightarrow \infty, \psi \rightarrow y$, remains but the upstream condition is now a matching condition with the flow on the other scale: $x \rightarrow-\infty, \psi \rightarrow \Psi(y)$.

The requirement for momentum conservation allows us to find $W_{\infty}$ without solving this problem fully. Using the present variables, momentum conservation may be written in the following form:

$$
I=\int_{0}^{1}\left[\frac{1}{2}\left(\psi_{y}^{2}-\psi_{x}^{2}\right)-\int_{0}^{\psi} \omega \mathrm{d} \psi\right] \mathrm{d} y=\text { constant },
$$

where, as usual, subscripts $x$ and $y$ denote differentiation. As this form is somewhat unusual it is worth noting that its validity is easy to verify by taking its derivative with respect to $x$ and using (2.1), as follows:

$$
\begin{aligned}
\frac{\mathrm{d} I}{\mathrm{~d} x} & =\int_{0}^{1}\left(\psi_{y} \psi_{x y}-\psi_{x} \psi_{x x}-\omega \psi_{x}\right) \mathrm{d} y=\int_{0}^{1}\left[\psi_{y} \psi_{x y}-\psi_{x} \psi_{x x}+\psi_{x}\left(\psi_{x x}+\psi_{y y}\right)\right] \mathrm{d} y \\
& =\int_{0}^{1}\left(\psi_{y} \psi_{x y}+\psi_{x} \psi_{y y}\right) \mathrm{d} y=\int_{0}^{1} \psi_{y} \psi_{x y} \mathrm{~d} y+\int_{0}^{1} \psi_{x} \mathrm{~d} \psi_{y} \\
& =\int_{0}^{1} \psi_{y} \psi_{x y} \mathrm{~d} y+\left.\psi_{x} \psi_{y}\right|_{0} ^{1}-\int_{0}^{1} \psi_{y} \psi_{x y} \mathrm{~d} y=0 .
\end{aligned}
$$

Far upstream and far downstream the vertical velocity component, $-\psi_{x}$, vanishes. Substituting in (3.3) $\omega$ from (2.1) and $\psi$ from (3.1) we obtain

$$
I=-\frac{1}{24} \Lambda_{\infty}^{2} W_{\infty}^{3}+\frac{1}{6} R i\left(1-W_{\infty}^{3}\right)+\frac{1}{2} R i^{\frac{1}{2}} W_{\infty}^{2} \cot \left[\left(1-W_{\infty}\right) R i^{\frac{1}{2}}\right]+\frac{1}{2}\left(1+W_{\infty}\right) .
$$




\begin{tabular}{clcccccc}
$\Gamma$ & $R i$ & $A$ & $C$ & $L_{\frac{1}{2}}$ & $W$ & $b$ & $\alpha$ \\
0 & 0 & $\infty$ & 73 & 0 & 0 & 0.55 & 0.44 \\
0.5 & 0 & 74.1 & 74 & 0.088 & 0.052 & 0.544 & 0.44 \\
2.5 & 0 & 17.0 & 85 & 0.409 & 0.244 & 0.541 & 0.442 \\
5 & 0 & 11.1 & 111 & 0.735 & 0.414 & 0.538 & 0.419 \\
10 & 0 & 9.27 & 186 & 1.23 & 0.572 & 0.527 & 0.355 \\
20 & 0 & 9.01 & 360 & 2.09 & 0.651 & 0.515 & 0.254 \\
40 & 0 & 9.00 & 720 & 3.76 & 0.666 & 0.508 & 0.157 \\
50 & 0 & 9.00 & 900 & 4.59 & 0.666 & 0.506 & 0.132 \\
$\infty$ & 0 & 9 & $\infty$ & $\infty$ & $\frac{2}{3}$ & $\frac{1}{2}$ & 0 \\
0.5 & 5.0 & 69.7 & 70 & 0.090 & 0.054 & 0.546 & 0.44 \\
2.5 & 5.0 & 11.1 & 57 & 0.507 & 0.301 & 0.541 & 0.440 \\
5 & 5.0 & 7.62 & 80 & 0.920 & 0.471 & 0.532 & 0.388 \\
10 & 5.0 & 7.05 & 149 & 1.56 & 0.572 & 0.519 & 0.292 \\
30 & 5.0 & 7.01 & 441 & 3.91 & 0.608 & 0.513 & 0.231 \\
$\infty$ & 5.0 & 7.01 & $\infty$ & $\infty$ & 0.608 & $\frac{1}{2}$ & 0 \\
0.5 & 8.5 & 63.0 & 64 & 0.095 & 0.056 & 0.544 & 0.44 \\
2.5 & 8.5 & 5.40 & 39 & 0.784 & 0.388 & 0.530 & 0.376 \\
5 & 8.5 & 4.72 & 71 & 1.42 & 0.468 & 0.516 & 0.262 \\
10 & 8.5 & 4.68 & 136 & 2.49 & 0.499 & 0.508 & 0.172 \\
15 & 8.5 & 4.69 & 201 & 3.55 & 0.503 & 0.505 & 0.127 \\
$\infty$ & 8.5 & 4.68 & $\infty$ & $\infty$ & 0.502 & $\frac{1}{2}$ & 0 \\
0.5 & 9.0 & 59.0 & 60 & 0.099 & 0.058 & 0.546 & 0.43 \\
2.5 & 9.0 & 4.40 & 41 & 0.946 & 0.389 & 0.522 & 0.317 \\
5 & 9.0 & 4.12 & 75 & 1.67 & 0.447 & 0.512 & 0.219 \\
10 & 9.0 & 4.11 & 141 & 2.97 & 0.465 & 0.506 & 0.138 \\
$\infty$ & 9.0 & 4.08 & $\infty$ & $\infty$ & 0.464 & $\frac{1}{2}$ & 0 \\
& & & TABLE 1.8 Basic results & & & \\
\hline
\end{tabular}

Far downstream the flow is uniform, $\psi=y$. This yields $I=\frac{1}{2}+\frac{1}{6} R i$. As the value of $I$ must be the same far upstream and far downstream we obtain

$$
-\frac{1}{24} \Lambda_{\infty}^{2} W_{\infty}^{3}-\frac{1}{6} R i W_{\infty}^{3}+\frac{1}{2} R i^{\frac{1}{2}} W_{\infty}^{2} \cot \left[\left(1-W_{\infty}\right) R i^{\frac{1}{2}}\right]+\frac{1}{2} W_{\infty}=0 .
$$

Together with (3.2) this gives two equations for the two unknowns, $A_{\infty}$ and $W_{\infty}$. These equations can be solved in a parametric form. Denote

$$
\lambda=\left(1-W_{\infty}\right) R i^{\frac{1}{2}}, \quad R i^{\frac{1}{2}}=\lambda /\left(1-W_{\infty}\right) .
$$

Substituting $\Lambda_{\infty}$ from (3.2) and $R i^{\frac{1}{2}}$ from (3.5) into (3.4) yields an equation for $W_{\infty}$ :

$$
\left[(1-\lambda \cot \lambda)^{2}+\lambda^{2}+3 \lambda \cot \lambda-3\right] W_{\infty}^{2}+(4-\lambda \cot \lambda) W_{\infty}-2=0 \text {. }
$$

This equation can be solved explicitly, with the correct choice between the two solutions made using the evident condition that $0 \leqslant W_{\infty} \leqslant 1$. The values of $R i$ and $A_{\infty}$ can then be found from (3.2) and (3.5). The functions $A_{\infty}(R i)$ and $W_{\infty}(R i)$ are plotted in Figure 2. Note that the limit $\lambda \rightarrow 0$ gives a non-stratified flow: $R i=0, A_{\infty}=9$, $W_{\infty}=\frac{2}{3}$. For $\lambda \rightarrow \pi, R i \rightarrow \pi^{2}, \Lambda_{\infty} \rightarrow 0$ and $W_{\infty} \rightarrow 0$. Hence, in this limit the long-eddy width tends to zero. For $R i=\pi^{2}$ momentum conservation allows two states of the flow with $W_{\infty}=0$ : a uniform one and that described by (3.1) with $W_{\infty}=0$. For finite eddies the numerical results given in table 1 show that the eddy width increases with the eddy length for a given $R i$, and hence the width of the finite eddy must tend to zero for $R i \rightarrow \pi^{2}$ also. Although this simplifies the asymptotic investigation of this limit, it remains a very difficult problem. 
Near the front and rear stagnation points this description breaks down. The solution of the full equation with corresponding boundary conditions is needed. From the computational point of view, this solution may be obtained as a solution of the original problem with very large $\Gamma$ (specified circulation round the eddy). The calculations with the largest $\Gamma$ described below are quite suitable for this purpose. Hence, the uniformly valid long-eddy asymptotics are in fact obtained.

\section{Numerical procedure}

The vertical eddy symmetry axis was chosen to be the left-hand boundary of the computational domain, and the right-hand boundary was located sufficiently far downstream at $x=x_{\varphi}$ and the condition $\psi=y$ applied at it. Equation (2.1) was replaced by

$$
\partial \psi / \partial t=\nabla^{2} \psi+\omega(\psi)
$$

The widely known alternating-direction method was used, with a finite-difference scheme which had second-order accuracy everywhere except in the close vicinity of the eddy boundary, where the second derivatives were approximated only to first order. Note that the very weak singularities near the stagnation points (see Sadovskii $1971 b$, or Saffman \& Tanveer 1982) cannot affect the overall accuracy of the computations. The time step was chosen to be 0.3 of an optimum time step for the corresponding linear problem with $R i=0$ in order to suppress the anticipated nonlinear effects. As the method proved to be very efficient no different choices were tried.

Considering $-\psi$ as a temperature, an equation like (4.1) with the boundary conditions corresponding to those in $\S 1$ describes heat conduction in a rectangular region, at the lower boundary of which the temperature is zero and negative at the right and upper ones. The left-hand boundary is thermally insulated. The source term then corresponds to local heating (note that $A>0$ because the circulation in the eddy is clockwise). For $R i=0$ it is only non-zero in the positive temperature region. The heating then is proportional to $\Lambda$ and the evident analogy with a sheet of paper set on fire shows that the anticipated solution with $A$ independent of $t$ can be unstable. For large $A$ almost the whole sheet will be consumed by fire, and for small $A$ the fire will go out because of the cooling at the walls. Analysis of the corresponding onedimensional problem (with $\psi$ depending only on $y$ and $t$ ) supports this idea to some extent. For these reasons no attempts were made to do calculations with constant $\boldsymbol{A}$. Instead, before each time step the area $S$ of the eddy was calculated and the new value of $A$ was determined as $A=\Gamma / S$ where $\Gamma$ is the specified circulation around the eddy. In this way if the region on fire (the eddy) shrinks, the heating increases and vice versa.

The usual criterion for slowly convergent iterations was applied. The iterations were stopped if $\left\|\psi^{n}-\psi^{n-1}\right\| \leqslant \epsilon(1-\lambda)$ with $\lambda=\left\|\psi^{n}-\psi^{n-1}\right\| /\left\|\psi^{n-1}-\psi^{n-2}\right\|$. Here $n$ is the iteration number and

$$
\left\|\psi^{n}\right\|=\left(\sum_{i j}\left(\psi_{i j}^{n}\right)^{2} \Delta x \Delta y\right)^{\frac{1}{2}}
$$

$\Delta x, \Delta y$ are the grid steps and $\epsilon$ is the anticipated accuracy.

The background to this criterion is as follows. Consider an iteration process $x_{n}=f\left(x_{n-1}\right)$ which converges to the solution $x$ of the equation $x=f(x)$. For $x_{n}$ sufficiently close to $x$ we have $x_{n}=x+f^{\prime}(x)\left(x_{n}-x\right)$. Hence $\left(x_{n}-x_{n-1}\right)=\left(f^{\prime}(x)-1\right)$ $\left(x_{n-1}-x\right)$. This means that the correct estimate of the accuracy achieved is

$$
\epsilon_{n-1} \approx\left|\left(x_{n}-x_{n-1}\right) /\left(f^{\prime}(x)-1\right)\right| .
$$


For slowly converging iterations $f^{\prime}(x)$ is close to 1 , so the widely used criterion, $\left|x_{n}-x_{n-1}\right|<\epsilon$, gives erroneous results. The value of $f^{\prime}(x)$ can be estimated via the last three iterations. The extension to a multi-dimensional vector $x$, together with the assumption that the major eigenvalue of the matrix of derivatives $\left\{\partial \psi_{i j}^{n} / \partial \psi_{k l}^{n-1}\right\}$ is real, leads directly to the criterion used here. More detailed discussion can be found in texts on numerical methods.

The total accuracy of the calculations depends on the size of the computational domain $x_{r}$, on the numbers of grid lines in the $x$ - and $y$-directions $(N$ and $M$, respectively), on $\epsilon$ and on the rounding errors $\left(10^{-8}\right.$ for single precision and $10^{-16}$ for double precision). The memory size of the computers used imposed the restriction $N \times M<30000$ for single-precision calculations. Assuming $N$ and $M$ to be of the same order of magnitude and taking into account the second order of accuracy of the numerical scheme we obtain a minimum error of about $1 / N^{2} \approx 10^{-4}$, with maximum usage of available memory. Assuming that the (single-precision) rounding errors are summed as independent random quantities and that the number of operations per time step is of order $N \times M$, the rounding errors per time step can be estimated as $(N \times M)^{\frac{1}{2}} \times 10^{-8} \approx 10^{-4}$. For double-precision calculations the estimate would be $10^{-12}$. However, the total error would be greater because of the inevitable reduction in the maximum possible $N \times M$. For this reason most calculations were made with single precision. The value of $\epsilon$ in the convergence criterion cannot be made much smaller than the rounding error per time step because of the random behaviour of the rounding error. Note that, owing to the iterative nature of the process, the rounding errors do not accumulate.

The influence of the different parameters on the accuracy of the calculations is illustrated by the following examples for $\Gamma=5, R i=2.5$. For $x_{r}=4, N=81, M=41$ and $\epsilon=10^{-3}, 10^{-4}$, and $10^{-5}$ the values of the eddy half-length $L_{\frac{1}{2}}$ (the most sensitive quantity) were $0.8093,0.8090$ and 0.8090 , respectively (rounded to four decimal places). For $\epsilon=5 \times 10^{-5}, M=41, N=20 x_{r}+1, x_{r}=3,4$ and 5 the values of $L_{\frac{1}{2}}$ were $0.8091,0.8089$ and 0.8086 . For $\epsilon=10^{-5}, x_{r}=2, N=2(M-1)+1, M=25,37,5^{2} 5$ and 82 the values of $L_{\frac{1}{2}}$ were $0.8093,0.8054,0.8044$ and 0.8039 . Tests of this kind were also done for other values of $\Gamma$ and $R i$. In view of these tests and the preceding considerations, the accuracy in most cases was estimated as $10^{-4}-10^{-5}$. To improve on this more computer memory would be needed. As only uniform grids were used in the calculations, the flows with small eddies (corresponding to small $\Gamma$ and large $R i$ ) were calculated less accurately because of insufficient resolution inside the eddies. Nevertheless, the errors in the data plotted here are within graphical accuracy and all the digits (with the final ones rounded, not truncated) in the table for $R i=0, \Gamma \neq 0$ are correct. Errors in the results for $R i>0$ in table are believed to be less than $1 \%$ (and much smaller in most cases) except for $R i=8.5$ and 9 with $\Gamma=0.5-2.5$ where they may be as large as $5 \%$.

Calculations for $\Gamma=0.5-2.5, R i=0$ were made on a $161 \times 161$ grid with $x_{r}=1$ and for $\Gamma=5-50, R i \geqslant 0$ on a $391 \times 61$ grid with $x_{r}=6$. For $R i>0$ and small $\Gamma$, grids of $241 \times 121$ and $301 \times 101$ nodes with $x_{r}=2$ and $x_{r}=3$ were used. Downstream of the eddy the expansion

$$
\psi=\sum_{n}^{\infty} A_{n} \sin (\pi n y) \exp \left(-\lambda_{n} x\right)
$$

is valid and $\lambda_{1}=\left(\pi^{2}-R i\right)^{\frac{1}{2}}$. Accordingly, for larger $R i$ longer computational domains were needed.

The correctness of the program was also checked by the comparison of the total 

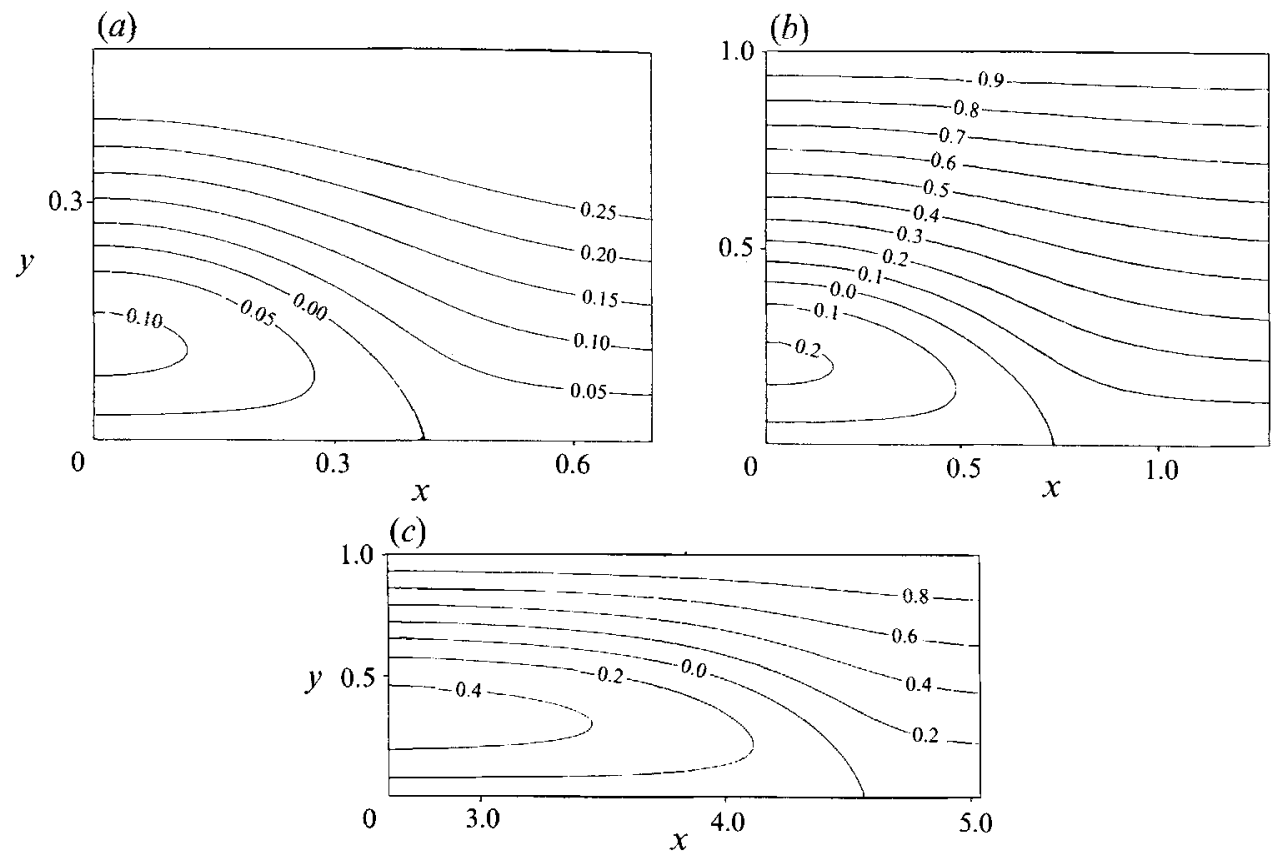

Figure 3. Streamlines for $R i=0$ for (a) $\Gamma=2.5,(b) \Gamma=5,(c) \Gamma=50$.

momentum flux through the left- and right-hand sides of the computational domain. It is also supported by the agreement with the independently calculated long-eddy asymptotics (see table 1) and by the agreement with the results of other authors (see below). Without undertaking the computations to a much higher degree of precision, it is not possible to be absolutely certain that these calculated solutions do, in fact, represent real solutions. However, the results of these various checks provided considerable confidence in this regard.

\section{Results}

Results are represented in table 1 and figures $3-5$. In the table, $C$ is the energy dissipation coefficient defined by $C=2 \iint \omega^{2} \mathrm{~d} x \mathrm{~d} y$ and $L_{1}$ is the half-length of the eddy. The quantities $b$, the ratio of the circulation over the upper part of the eddy boundary to the circulation around the whole eddy, and $\alpha$, the ratio of the common area of both (upper and lower) halves of the eddy to the square of the eddy length, are included because of their role in the asymptotic theory of the corresponding viscous flows. In figure $3(a)$ the streamlines for $\Gamma=2.5$ and $R i=0$ are shown. The value of $\Gamma$ is sufficiently small for the influence of the upper wall on the eddy shape to be negligible. In figure $3(b)$ the streamlines for $\Gamma=5$ are shown and in figure $3(c)$ the streamlines for a very long eddy $(\Gamma=50, R i=0)$ near the eddy end are shown. Figure 4 shows the change of the eddy shape with the Richardson number.

Figure 5 shows the change in eddy shape with $\Gamma$ for $R i=0$. Sadovskii's (1970) results, taken from the table in his paper, and Moore, Saffman \& Tanveer's (1988) results, taken from their plot, are shown. Since the decrease of the Bernoulli constant on the dividing streamline equals zero in the present calculation, only the corresponding results of these other authors are included and no comparison with the results of Smith (1986) is possible. Our results are restricted to $\Gamma \neq 0$, while Sadovskii's and Moore 


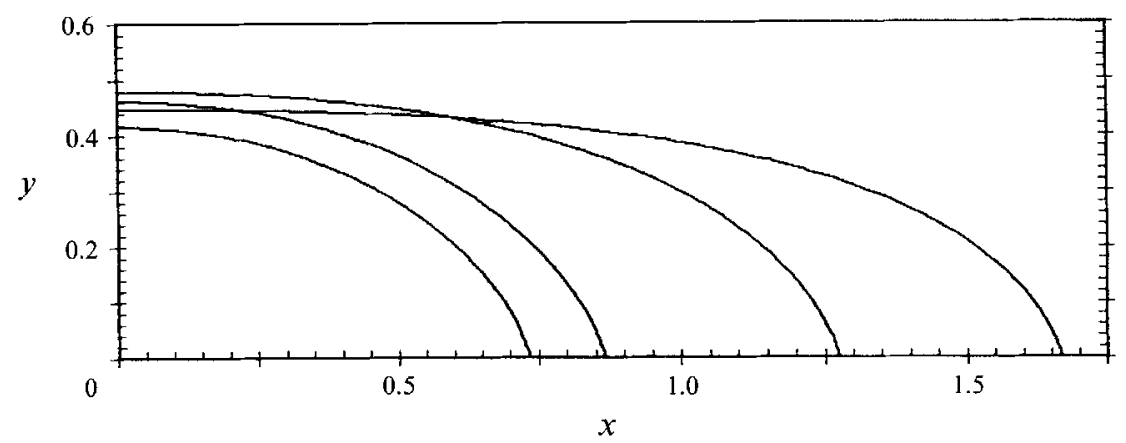

Figure 4. The eddy shape for $\Gamma=5, R i=0,4,8,9$. The eddy length grows with $R i$.

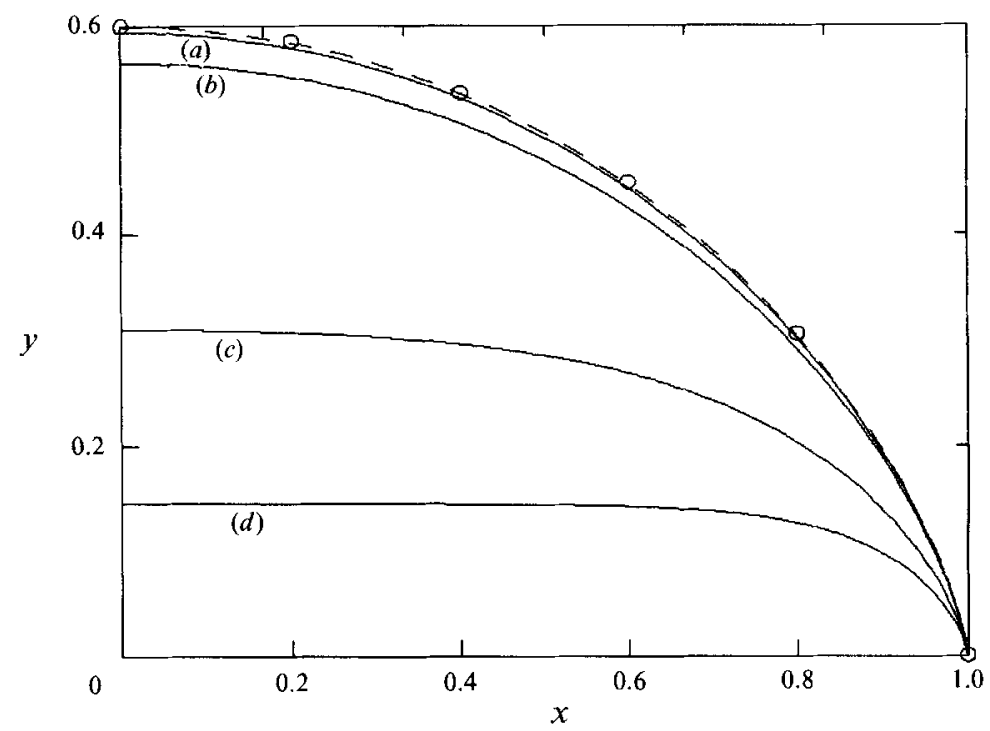

FIGURE 5. Comparisons of the eddy shape for $R i=0$. The lengthscale is the eddy length. Solid curves, present work: (a) $\Gamma=2.5$; (b) $\Gamma=5$; (c) $\Gamma=20$; (d) $\Gamma=50$. Dashed curve, Sadovskii (1970), $\Gamma=0$. O, Moore et al. (1988), $\Gamma=0$.

et al.'s results are for $\Gamma=0$ only. Results for $\Gamma=0.5$ and 1.0 are not shown as they are indistinguishable from the Sadovskii result. All results (for sufficiently small $\Gamma$ ) are in excellent agreement. It should be noted that the discrepancy between Sadovskii's and Moore et al.'s values of $A L_{\frac{1}{2}}$ ( $\omega$ in the notation of Moore et al.) in the case of zero Bernoulli decrease is due to an error in the handling of computed data in the former work. This was corrected later (Sadovskii \& Kozhuro 1977). The corrected Sadovskii value of 6.5 is in good agreement with that of Moore et al. and extrapolation of the present results to the $\Gamma=0$ case yields the value 6.44 .

Results for $\Gamma=0$ in table 1 correspond to the unbounded flow. They were obtained by an extrapolation from calculated solutions for $R i=0$, and $\Gamma=0.5$ and 1.0 . These results are in reasonable agreement with the results of other authors. Results for $R i>0$ must approach those for $R i=0$ when $\Gamma \rightarrow 0$. This tendency is clearly seen in the table. Results for $\Gamma=\infty$ in the table were obtained from the long-eddy asymptotics given in $\S 3$.

The behaviour of the solution as a function of $R i$ is complicated. For example, figure 4 shows a non-monotonic dependence of $W$ on $R i$ for $\Gamma=5$. The asymptotics for 
$R i \rightarrow \pi^{2}$ were not investigated here though this could contribute considerably to the understanding of the flow.

For $R i>\pi^{2}$ wave disturbances must appear (see Turner 1973, for example, for a discussion of the extensive literature on such cases). A symmetry imposed in our calculations implies in this case a symmetric wave upstream and downstream of the eddy. Because of this symmetry there would be no wave drag. Such flows seem to be of no interest, since they would generally be quite unphysical.

This work was undertaken mainly during a visit to the University of Surrey, England and was financially supported by the Royal Society of London. The author is very grateful to Professor I. P. Castro who drew his attention to stratified flows and also for numerous comments, discussions and textual improvements, to $\mathrm{Dr} \mathrm{K}$. N. Atkinson whose programs were used to prepare the data for plotting and to the whole staff of the Department of Mechanical Engineering of the University of Surrey for their hospitality and friendliness.

\section{REFERENCES}

Chernyshenko, S. I. 1988 The asymptotics of the steady separated flow past a body at large Reynolds number. Appl. Math. Mech. 52, 746.

MoOre, D. W., Saffman, P. G. \& TANVEer, S. 1988 The calculation of some Batchelor flows: the Sadovskii vortex and rotational corner flow. Phys. Fluids 31, 978.

Pierrehumbert, R. T. 1980 A family of steady, translating vortex pairs with distributed vorticity. J. Fluid Mech. 99, 129.

SADOVSKII, V.S. 1970 A region of uniform vorticity in plane potential flow. Uch. Zap. TSAGI 1, 1 (in Russian).

SADOVSKII, V.S. 1971 a Vortex regions in a potential stream with a jump of Bernoulli's constant at the boundary. Appl. Math. Mech. 35, 729.

SADOvSKII, V. S. $1971 b$ Properties of potential and vortex flows touching at a closed streamline. Uch. Zap. Ts AGI 2, 113 (in Russian).

SadovskiI, V. S. \& Kozhuro, L. A. 1977 Two one-parameter families of inviscid vortex flows. ChMMSS 8, 126 (in Russian).

Saffman, P. G. \& TAnveer, S. 1982 The touching pair of equal and opposite vortices. Phys. Fluids 25, 1929.

SMITH, F. T. 1986 Concerning inviscid solutions for large-scale separated flows. J. Engng Maths 20, 271.

TURfus, C. 1993 Prandtl-Batchelor flow past a flat plate at normal incidence in a channel-inviscid analysis. J. Fluid Mech. 249, 59.

TURNER, J. S. 1973 Buoyancy effects in Fluids. Cambridge University Press. 